\title{
A THREE-PHASE FLOW MODEL WITH TWO MISCIBLE PHASES
}

\author{
J.-M. HÉRARD AND H. MATHIS
}

\begin{abstract}
The paper concerns the modelling of a compressible mixture of a liquid, its vapor and a gas. The gas and the vapor are miscible while the liquid is immiscible with the gaseous phases. This assumption leads to non symmetric constraints on the void fractions. We derive a three-phase threepressure model endowed with an entropic structure. We show that interfacial pressures are uniquely defined and propose entropy-consistent closure laws for the source terms. Naturally one exhibits that the mechanical relaxation complies with Dalton's law on the phasic pressures. Then the hyperbolicity and the eigenstructure of the homogeneous model are investigated and we prove that it admits a symmetric form leading to a local existence result. We also derive a barotropic variant which possesses similar properties.
\end{abstract}

Key-words. Multi-component compressible flows, entropy, relaxation, phase transition, miscibility constraint, closure conditions, hyperbolicity.

2010 MCS. 76T30, 35L60, 35Q35.

\section{Contents}

1. Introduction

2. A three-velocity three-pressure relaxation model 3

2.1. Definition of the interfacial pressures 4

2.2. Nature of the convective system 6

2.3. Admissible structure of the source terms 9

2.4. Symmetrization 11

3. Conclusion 13

Appendix A. Phasic equations 14

Appendix B. Derivation of a barotropic model $\quad 15$

References

\section{INTRODUCTION}

The present paper concerns the modelling of multiphase compressible flows which arise in many industrial applications. For instance, in the framework of safety demonstration for pressurized water reactors, some scenarii involve compressible three-phase flows. This is the case for the Loss Of Coolant Accident (LOCA) when a brutal rupture in the coolant circuit creates phase transition waves in the system

Date: January 7, 2019. 
and leads to the appearance of vapor inside the liquid, the both phases interacting with the ambiant air. It also happens in Reactivity Initiated Accident (RIA) which involves liquid water and its vapor combined with hot fission gases, see IRSN website. It is then crucial to propose a model accounting for the dynamical and the thermodynamical disequilibrium (with respect to pressure, temperature, chemical potential). Over the last twenty years, a vast literature about the modeling of multiphase flows, but above all of two-phase flows, has been developed. Most of these works rely on the two-fluid approach [1, 9, 8, 10, 25,4]: the dynamic of each phase is described by an Euler-type model and the two systems are coupled through interfacial (nonconservative) terms and source terms which relax towards the thermodynamical equilibrium. Different extensions have been proposed in the two-phase framework, see for instance $[5,28,27]$ for recent contributions. The analysis of such models has been investigated in [6] (entropy structure, symmetrization, local existence result) and [11] (hierarchy of relaxation models and subcharacteristic condition). The generalization to multicomponent mixtures has been the subject of recent contributions: see [30, 18] for the modelling and the analysis of proper closure laws, [21] for the barotropic restriction, and [23, 3, 17] for the modelling of chemical exchanges between the components. Another approach consists in considering the mixture as one fluid (thus with a unique velocity for all the phases) with appropriate thermodynamical properties. This type of model is referred as an homogeneous model, see [2] for a two-phase case and [22] for a three-phase extension.

Note that in the previous references, the phases are assumed to be immiscible in the sense that all components occupy different volumes at a mesoscopic scale. In [7] a multicomponent Euler type model is proposed for a fully miscible mixture of $k$ phases, as the $k$ phases are perfectly intricate and occupy the whole volume.

The present work focuses on the modeling of a three-phase mixture of a liquid (with index $l$ ), its vapor (with index $v$ ) and a gas (with index $g$ ). The gas and the vapor are supposed to be miscible in the sense that they are perfectly intimate like ideal gases. This leads to the equality of the phasic void fractions of vapor and gas, for any $(t, x) \in \mathbb{R}^{+} \times \mathbb{R}$

$$
\alpha_{v}(t, x)=\alpha_{g}(t, x) \in[0,1] .
$$

Conversely the liquid is immiscible with the gaseous phases in the sense that

$$
\alpha_{l}(t, x)+\alpha_{g}(t, x)=1,
$$

with $\alpha_{l}(t, x) \in[0,1]$ and

$$
\partial_{x} \alpha_{l}(t, x)=-\partial_{x} \alpha_{g}(t, x), \quad \text { and } \quad \partial_{x} \alpha_{v}(t, x)=\partial_{x} \alpha_{g}(t, x) .
$$

Mass transfer may occur between the vapor and the liquid but no phase transition is allowed between the gas and the two remaining phases, leading to a constant mass of gas in the system. The derivation of an homogeneous model for such a three-phase mixture has been investigated in [29]. In the present work we address the derivation of a three-fluid model, in the spirit of [19], but accounting for the volume constraint (1)-(2).

We do not tackle the numerical approximation of such systems and refer to [24] and references therein in the two-fluid framework, in [32, 31] for immiscible three-phase mixture case and to [16] for the multicomponent case. 


\section{A THREE-VELOCity THREE-PRESSURE RELAXATION MODEL}

The compressible flow is composed of a liquid phase, with index $l$, its vapor, with index $v$ and a gaseous phase, with index $g$. For sake of readability we define the set of indices $\mathbb{K}:=\{l, g, v\}$. Defining the state vector $\mathbf{Y}$ as

$$
\mathbf{Y}=\left(\alpha_{g}, \rho_{l}, \rho_{g}, \rho_{v}, u_{l}, u_{g}, u_{v}, \varepsilon_{l}, \varepsilon_{g}, \varepsilon_{v}\right) \in \mathbb{R}^{10}
$$

we consider the following governing set of equations:

$$
\begin{array}{ll}
\partial_{t} \alpha_{g}+v_{i}(\mathbf{Y}) \partial_{x} \alpha_{g}=\Phi_{g}(\mathbf{Y}), & \\
\partial_{t} m_{k}+\partial_{x}\left(m_{k} u_{k}\right)=\Gamma_{k}(\mathbf{Y}), & k \in \mathbb{K}, \\
\partial_{t}\left(m_{k} u_{k}\right)+\partial_{x}\left(m_{k} u_{k}^{2}+\alpha_{k} p_{k}\right)+\pi_{k}(\mathbf{Y}) \partial_{x} \alpha_{g}=\mathcal{D}_{k}(\mathbf{Y}), & k \in \mathbb{K}, \\
\partial_{t}\left(m_{k} E_{k}\right)+\partial_{x}\left(u_{k}\left(m_{k} E_{k}+\alpha_{k} p_{k}\right)\right)-\pi_{k}(\mathbf{Y}) \partial_{t} \alpha_{g}=\mathcal{B}_{k}(\mathbf{Y}) & k \in \mathbb{K} .
\end{array}
$$

where $\alpha_{k}$ is the void fraction of the phase $k=l, g, v$ and $m_{k}=\alpha_{k} \rho_{k}$ is the partial mass of the phase $k \in \mathbb{K}$ with $\rho_{k}$ the mean phasic density. The pressure within the phase $k \in \mathbb{K}$ is denoted $p_{k}$. Each phase evolves with its own velocity $u_{k}, k \in \mathbb{K}$, and the phasic total energies $E_{k}$ read

$$
E_{k}=u_{k}^{2} / 2+\varepsilon_{k}
$$

where $\varepsilon_{k}=\varepsilon_{k}\left(p_{k}, \rho_{k}\right)$ is the phasic internal energy. The phasic specific entropy $s_{k}$ is a function of the phasic pressure $p_{k}$ and density $\rho_{k}$. The phasic speed of sound $c_{k}$ complies with

$$
c_{k}^{2} \frac{\partial s_{k}}{\left.\partial p_{k}\right|_{\rho_{k}}}+\frac{\partial s_{k}}{\left.\partial \rho_{k}\right|_{p_{k}}}=0
$$

and

$$
\rho_{k} c_{k}^{2}=\left(\frac{\partial \varepsilon_{k}}{\partial p_{k}}\right)^{-1}\left(\frac{p_{k}}{\rho_{k}}-\rho_{k} \frac{\partial \varepsilon_{k}}{\partial \rho_{k}}\right) .
$$

We also define the phasic temperature

$$
a_{k}=\frac{\partial s_{k}}{\partial p_{k}}\left(\frac{\partial \varepsilon_{k}}{\partial p_{k}}\right)^{-1} \geq 0
$$

the phasic enthalpies $h_{k}$ and chemical potentials $\mu_{k}$

$$
\begin{aligned}
& h_{k}=\varepsilon\left(p_{k}, \rho_{k}\right)+p_{k} / \rho_{k}, \quad H_{k}=E_{k}+p_{k} / \rho_{r}, \\
& \mu_{k}=h_{k}-a_{k}^{-1} s_{k}\left(p_{k}, \rho_{k}\right) .
\end{aligned}
$$

We denote $\mathbf{U} \in \mathbb{R}^{10}$ the vector of "conservative" variables defined by

$$
\mathbf{U}=\left(\alpha_{g}, \mathbf{u}_{l}, \mathbf{u}_{g}, \mathbf{u}_{v}\right) \in \mathbb{R}^{10} \text {, with } \mathbf{u}_{k}=\left(m_{k}, m_{k} u_{k}, m_{k} E_{k}\right) \in \mathbb{R}^{3}, k \in \mathbb{K} .
$$

Equations (5), (7) and (8) involve nonconservative terms with the interfacial velocity $v_{i}(\mathbf{Y})$ and the interfacial pressures $\pi_{k}(\mathbf{Y}), k \in \mathbb{K}$. The definition of the velocity $v_{i}(\mathbf{Y})$ will be addressed in the following section. The interface quantities $\pi_{k}(\mathbf{Y})$ should cancel each other out in the sense that

$$
\sum_{k=l, g, v} \pi_{k}(\mathbf{Y})=0
$$

Similarly the source terms cancel when considering the mean flow, that is

$$
\sum_{k \in \mathbb{K}} \Gamma_{k}(\mathbf{Y})=0, \quad \sum_{k \in \mathbb{K}} \mathcal{D}_{k}(\mathbf{Y})=0, \quad \sum_{k \in \mathbb{K}} \mathcal{B}_{k}(\mathbf{Y})=0 .
$$


Note that the equation (5) involves only the source term $\Phi_{g}(\mathbf{Y})$ associated to the void fraction $\alpha_{g}$. Nonetheless one should consider the mechanical transfer terms on the two remaining phases $k=l, v$ so that

$$
\Phi_{g}(\mathbf{Y})=\Phi_{v}(\mathbf{Y})=-\Phi_{l}(\mathbf{Y})
$$

2.1. Definition of the interfacial pressures. Using Galilean invariance, we postulate that the interfacial velocity takes the form:

$$
v_{i}(\mathbf{Y})=\beta_{l}(\mathbf{Y}) u_{l}+\beta_{g}(\mathbf{Y}) u_{g}+\beta_{v}(\mathbf{Y}) u_{v},
$$

with

$$
\left\{\begin{array}{l}
\beta_{l}(\mathbf{Y})+\beta_{g}(\mathbf{Y})+\beta_{v}(\mathbf{Y})=1, \\
\beta_{k}(\mathbf{Y}) \geq 0, \quad k \in \mathbb{K} .
\end{array}\right.
$$

Note that other definitions of the interfacial velocity exist in the literature, see [15] for a review and a comparison of the most common definitions. Less standard definitions may be given, see for instance [5] where the quantities $\beta_{k}(\mathbf{Y})$ follow a nonlinear transport equation.

Thus the relative velocity of the phase $k \in \mathbb{K}$ with respect to the interface reads

$$
v_{i}(\mathbf{Y})-u_{k}=\beta_{k^{\prime}}(\mathbf{Y})\left(u_{k^{\prime}}-u_{k}\right)+\beta_{k^{\prime \prime}}(\mathbf{Y})\left(u_{k^{\prime \prime}}-u_{k}\right),
$$

with $k, k^{\prime}, k^{\prime \prime} \in \mathbb{K}, k^{\prime} \neq k, k^{\prime \prime} \neq k, k^{\prime \prime} \neq k^{\prime}$. The relative velocities also satisfy the following useful identity

$$
u_{l}-u_{g}=\left(u_{l}-u_{v}\right)-\left(u_{g}-u_{v}\right) .
$$

The modelling of the interfacial quantities requires the definition of an entropy function for the system (5)-(8).

The mixture entropy is

$$
\eta(\mathbf{Y})=\sum_{k \in \mathbb{K}} m_{k} s_{k},
$$

and one can prove that smooth solutions of (5)-(8) satisfy the following entropy identity

$$
\partial_{t} \eta(\mathbf{Y})+\partial_{x} f_{\eta}(\mathbf{Y})+\mathcal{A}_{\eta}\left(\mathbf{Y}, \partial_{x} \mathbf{Y}\right)=R H S_{\eta}(\mathbf{Y}),
$$

where the entropy flux reads

$$
f_{\eta}(\mathbf{Y})=\sum_{k \in \mathbb{K}} m_{k} s_{k} u_{k}
$$

and

$$
\begin{aligned}
\mathcal{A}_{\eta}\left(\mathbf{Y}, \partial_{x} \mathbf{Y}\right)= & \sum_{k \in \mathbb{K}} a_{k}\left(v_{i}(\mathbf{Y})-u_{k}\right)\left(\pi_{k}(\mathbf{Y}) \partial_{x} \alpha_{g}+p_{k} \partial_{x} \alpha_{k}\right) \\
= & {\left[a_{v}\left(v_{i}(\mathbf{Y})-u_{v}\right)\left(\pi_{v}(\mathbf{Y})+p_{v}\right)+a_{g}\left(v_{i}(\mathbf{Y})-u_{g}\right)\left(\pi_{g}(\mathbf{Y})+p_{g}\right)\right.} \\
& \left.\left.\quad+a_{l}\left(v_{i}(\mathbf{Y})-u_{l}\right)\left(\pi_{l}(\mathbf{Y})-p_{l}\right)\right)\right] \partial_{x} \alpha_{g}
\end{aligned}
$$


and

$$
\begin{aligned}
R H S_{\eta} & =R H S_{\eta}^{\Phi} \\
& +\sum_{k \in \mathbb{K}} a_{k}\left(\mathcal{B}_{k}(\mathbf{Y})-u_{k} \mathcal{D}_{k}(\mathbf{Y})\right) \\
& +\sum_{k \in \mathbb{K}} a_{k} \Gamma_{k}(\mathbf{Y})\left(\frac{u_{k}^{2}}{2}-\varepsilon_{k}-\rho_{k} \partial_{\rho_{k}} \varepsilon_{k}\right) \\
& +\sum_{k \in \mathbb{K}} \Gamma_{k}(\mathbf{Y})\left(s_{k}+\rho_{k} \partial_{\rho_{k}} s_{k}\right),
\end{aligned}
$$

with

$$
\begin{aligned}
R H S_{\eta}^{\Phi} & =\sum_{k \in \mathbb{K}} a_{k}\left(\pi_{k}(\mathbf{Y}) \phi_{g}(\mathbf{Y})+p_{k} \Phi_{k}(\mathbf{Y})\right) \\
& =\left(a_{l}\left(\pi_{l}(\mathbf{Y})-p_{l}\right)+a_{g}\left(\pi_{g}(\mathbf{Y})+p_{g}\right)+a_{v}\left(\pi_{v}(\mathbf{Y})+p_{v}\right)\right) \Phi_{g}(\mathbf{Y}) .
\end{aligned}
$$

One obtains the entropy equation (23) by deriving the evolution equations on the phasic quantities, see details in Appendix A. The model with minimal entropy dissipation refers to the model (5)-(8) where $A_{\eta}\left(\mathbf{Y}, \partial_{x} \mathbf{Y}\right)=0$.

Proposition 1 (Definition of the interfacial pressures). Smooth solutions of the system (5)-(8) comply with the minimal entropy dissipation constraint $\mathcal{A}_{\eta}\left(\mathbf{Y}, \partial_{x} \mathbf{Y}\right)=0$ for the unique 3-tuple $\left(\pi_{v}(\mathbf{Y}), \pi_{g}(\mathbf{Y}), \pi_{l}(\mathbf{Y})\right)$, solution of

$$
\mathbb{C}\left(\pi_{l}(\mathbf{Y})-p_{l}, \pi_{g}(\mathbf{Y})+p_{g}, \pi_{v}(\mathbf{Y})+p_{v}\right)^{\top}=\left(0,0, p_{v}+p_{g}-p_{l}\right)^{\top},
$$

with

$$
\mathbb{C}=\left(\begin{array}{ccc}
-a_{l}\left(\beta_{g}(\mathbf{Y})+\beta_{v}(\mathbf{Y})\right) & a_{g} \beta_{l}(\mathbf{Y}) & a_{v} \beta_{l}(\mathbf{Y}) \\
a_{l} \beta_{g}(\mathbf{Y}) & -a_{g}\left(\beta_{l}(\mathbf{Y})+\beta_{v}(\mathbf{Y})\right) & a_{v} \beta_{g}(\mathbf{Y}) \\
1 & 1 & 1
\end{array}\right)
$$

Proof. Using the relations (20) and (21) on the relative velocities, the entropy dissipation $A\left(\mathbf{Y}, \partial_{x} \mathbf{Y}\right)$ reads also

$$
A\left(\mathbf{Y}, \partial_{x} \mathbf{Y}\right)=\left(u_{l}-u_{v}\right) \Theta_{l v}(\mathbf{Y})+\left(u_{g}-u_{v}\right) \Theta_{g v}(\mathbf{Y}),
$$

with

$$
\begin{aligned}
\Theta_{l v}(\mathbf{Y})= & -a_{l}\left(\beta_{g}(\mathbf{Y})+\beta_{v}(\mathbf{Y})\right)\left(\pi_{l}(\mathbf{Y})-p_{l}\right) \\
& +a_{g} \beta_{l}(\mathbf{Y})\left(\pi_{g}(\mathbf{Y})+p_{g}\right)+a_{v} \beta_{l}(\mathbf{Y})\left(\pi_{v}(\mathbf{Y})+p_{v}\right) \\
\Theta_{g v}(\mathbf{Y})= & a_{l} \beta_{g}(\mathbf{Y})\left(\pi_{l}(\mathbf{Y})-p_{l}\right) \\
& -a_{g}\left(\beta_{l}(\mathbf{Y})+\beta_{v}(\mathbf{Y})\right)\left(\pi_{g}(\mathbf{Y})+p_{g}\right)+a_{v} \beta_{g}(\mathbf{Y})\left(\pi_{v}(\mathbf{Y})+p_{v}\right) .
\end{aligned}
$$

Since the relative velocities are independant, canceling the entropy dissipation $A\left(\mathbf{Y}, \partial_{x} \mathbf{Y}\right)$ reduces to impose

$$
\Theta_{l v}(\mathbf{Y})=0, \quad \Theta_{g v}(\mathbf{Y})=0 .
$$

Now remind that the interfacial pressures $\pi_{k}(\mathbf{Y})$ cancel each other according to (15). Thus one can write the following identity

$$
\pi_{l}(\mathbf{Y})-p_{l}+\pi_{g}(\mathbf{Y})+p_{g}+\pi_{v}(\mathbf{Y})+p_{v}=p_{v}+p_{g}-p_{l}
$$

and one can rewrite the equations (32) and (33) under the linear system (28). The matrix $\mathbb{C}$ determinant is

$$
\operatorname{det}(\mathbb{C})=a_{l} a_{g} \beta_{v}(\mathbf{Y})+a_{l} a_{v} \beta_{g}(\mathbf{Y})+a_{g} a_{v} \beta_{l}(\mathbf{Y})
$$


Hence the system is invertible as soon as the quantities $a_{k}$ and $\beta_{k}(\mathbf{Y})$ are strictly positive. by

It follows that the vector $\left(\pi_{l}(\mathbf{Y})-p_{l}, \pi_{g}(\mathbf{Y})+p_{g}, \pi_{v}(\mathbf{Y})+p_{v}\right)^{\top}$ is uniquely defined

$$
\begin{aligned}
\left(\pi_{l}(\mathbf{Y})-p_{l}, \pi_{g}(\mathbf{Y})\right. & \left.+p_{g}, \pi_{v}(\mathbf{Y})+p_{v}\right)^{\top} \\
& =\frac{p_{v}+p_{g}-p_{l}}{\operatorname{det}(\mathbb{C})}\left(a_{g} a_{v} \beta_{l}(\mathbf{Y}), a_{v} a_{l} \beta_{g}(\mathbf{Y}), a_{l} a_{g} \beta_{v}(\mathbf{Y})\right)^{\top}
\end{aligned}
$$

As a consequence, using (35) and the constraint (19) on the quantities $\beta_{k}(\mathbf{Y})$, the term $R H S_{\eta}^{\Phi}$ given by (27) rewrites

$$
\begin{aligned}
R H S_{\eta}^{\Phi} & =\left(a_{l}, a_{g}, a_{v}\right)\left(\begin{array}{c}
\pi_{l}(\mathbf{Y})-p_{l} \\
\pi_{g}(\mathbf{Y})+p_{g} \\
\pi_{v}(\mathbf{Y})+p_{v}
\end{array}\right) \Phi_{g}(\mathbf{Y}) \\
& =\frac{p_{v}+p_{g}-p_{l}}{\operatorname{det}(\mathbb{C})} a_{l} a_{g} a_{v} \Phi_{g}(\mathbf{Y}) .
\end{aligned}
$$

For instance, if one imposes $v_{i}(\mathbf{Y})=u_{l}$ with $\beta_{l}(\mathbf{Y})=1$ and $\beta_{g}(\mathbf{Y})=\beta_{v}(\mathbf{Y})=0$ in (35) and (34), one ends up with

$$
\pi_{l}(\mathbf{Y})=p_{g}+p_{v}, \quad \pi_{g}(\mathbf{Y})=-p_{g}, \quad \pi_{v}(\mathbf{Y})=-p_{v}
$$

Here we consider that the interfacial pressures depend only on the state vector $\mathbf{Y}$ but one may consider another formulation, see [20] for instance for a dissipative formulation where the interfacial pressures are functions of $\mathbf{Y}$ and $\partial_{x} \mathbf{Y}$.

2.2. Nature of the convective system. It is now mandatory to determine an admissible closure law for the interfacial velocity $v_{i}(\mathbf{Y})$. When the three phasic pressures are equal and uniform (in space), the void fraction of gas $\alpha_{g}$ must be perfectly advected without smearing. Hence the field associated to the interfacial velocity $v_{i}(\mathbf{Y})$ must be linearly degenerated. To guarantee this property within the framework of two-phase flow models, it is rather classical to fix $v_{i}(\mathbf{Y})=u_{k}, k \in \mathbb{K}$, see $[14,12]$ for instance; we also refer to [15] for a comparison of other possible choices of interfacial velocities and their consequences.

In the sequel we consider arbitrarily $v_{i}(\mathbf{Y})=u_{l}$, thus the interfacial pressures $\pi_{k}(\mathbf{Y}), k \in \mathbb{K}$, are defined by (37).

The purpose of this section is to investigate the eigenstructure of the convective system and its hyperbolicity. We consider the state vector

$$
\mathbf{W}=\left(\alpha_{g}, p_{g}, u_{g}, p_{v}, u_{v}, p_{l}, u_{l}, s_{g}, s_{v}, s_{l}\right) \in \mathbb{R}^{10} .
$$

In the sequel we consider $\alpha_{l} \alpha_{g} \neq 0$.

Focusing on smooth solutions, the system (5)-(8) may rewrite under the quasilinear form

$$
\partial_{t} \mathbf{W}+\mathbb{B}(\mathbf{W}) \partial_{x} \mathbf{W}=0,
$$


with

$$
\mathbb{B}(\mathbf{W})=\left(\begin{array}{cccccccccc}
u_{l} & 0 & 0 & 0 & 0 & 0 & 0 & 0 & 0 & 0 \\
A_{g} & u_{g} & \rho_{g} c_{g}^{2} & 0 & 0 & 0 & 0 & 0 & 0 & 0 \\
0 & \tau_{g} & u_{g} & 0 & 0 & 0 & 0 & 0 & 0 & 0 \\
A_{v} & 0 & 0 & u_{v} & \rho_{v} c_{v}^{2} & 0 & 0 & 0 & 0 & 0 \\
0 & 0 & 0 & \tau_{v} & u_{v} & 0 & 0 & 0 & 0 & 0 \\
0 & 0 & 0 & 0 & 0 & u_{l} & \rho_{l} c_{l}^{2} & 0 & 0 & 0 \\
A_{l} & 0 & 0 & 0 & 0 & \tau_{l} & u_{l} & 0 & 0 & 0 \\
0 & 0 & 0 & 0 & 0 & 0 & 0 & u_{g} & 0 & 0 \\
0 & 0 & 0 & 0 & 0 & 0 & 0 & 0 & u_{v} & 0 \\
0 & 0 & 0 & 0 & 0 & 0 & 0 & 0 & 0 & u_{l}
\end{array}\right)
$$

where

$$
A_{l}=\frac{p_{g}+p_{v}-p_{l}}{m_{l}}, \quad A_{g}=\frac{\rho_{g} c_{g}^{2}}{\alpha_{g}}\left(u_{g}-u_{l}\right), \quad A_{v}=\frac{\rho_{v} c_{v}^{2}}{\alpha_{g}}\left(u_{v}-u_{l}\right) .
$$

Proposition 2 (Eigenstructure). The homogeneous system (39)-(41) is hyperbolic with real eigenvalues $\lambda_{1}=u_{g}, \lambda_{2}=u_{v}, \lambda_{3}=\lambda_{4}=u_{l}, \lambda_{5,6}=u_{g} \pm c_{g}, \lambda_{7,8}=u_{v} \pm c_{v}$, $\lambda_{9,10}=u_{l} \pm c_{l}$. Associated right eigenvectors span the whole space $\mathbb{R}^{10}$ except when some eigenvalues coincide, more precisely

$$
\left(u_{g}-u_{l}\right)^{2}-c_{g}^{2}=0, \quad \text { or } \quad\left(u_{v}-u_{l}\right)^{2}-c_{v}^{2}=0 .
$$

Note that the hyperbolicity is non-strict.

Proof. The matrix $\mathbb{B}(\mathbf{W})$ admits the following right eigenvectors

$$
\begin{aligned}
\mathbf{r}_{1}^{\top} & =(0,0,0,0,0,0,0,1,0,0), \\
\mathbf{r}_{2}^{\top} & =(0,0,0,0,0,0,0,0,1,0), \\
\mathbf{r}_{3}^{\top} & =(0,0,0,0,0,0,0,0,0,1), \\
\mathbf{r}_{4}^{\top} & =\left(1, A, B, C, D,-\rho_{l} A_{l}, 0,0,0,0\right), \\
\mathbf{r}_{5}^{\top} & =\left(0,-\rho_{g} c_{g}, 1,0,0,0,0,0,0,0\right), \\
\mathbf{r}_{6}^{\top} & =\left(0, \rho_{g} c_{g}, 1,0,0,0,0,0,0,0\right), \\
\mathbf{r}_{7}^{\top} & =\left(0,0,0,-\rho_{v} c_{v}, 1,0,0,0,0,0\right), \\
\mathbf{r}_{8}^{\top} & =\left(0,0,0, \rho_{v} c_{v}, 1,0,0,0,0,0\right), \\
\mathbf{r}_{9}^{\top} & =\left(0,0,0,0,0,-\rho_{l} c_{l}, 1,0,0,0\right), \\
\mathbf{r}_{10}^{\top} & =\left(0,0,0,0,0, \rho_{l} c_{l}, 1,0,0,0\right),
\end{aligned}
$$

where

$$
\left(\begin{array}{l}
A \\
B
\end{array}\right)=\frac{1}{\left(u_{g}-u_{l}\right)^{2}-c_{g}^{2}}\left(\begin{array}{cc}
u_{g}-u_{l} & -\rho_{g} c_{g}^{2} \\
-\tau_{g} & u_{g}-u_{l}
\end{array}\right)\left(\begin{array}{c}
-A_{g} \\
0
\end{array}\right)
$$

and

$$
\left(\begin{array}{l}
C \\
D
\end{array}\right)=\frac{1}{\left(u_{v}-u_{l}\right)^{2}-c_{v}^{2}}\left(\begin{array}{cc}
u_{v}-u_{l} & -\rho_{v} c_{v}^{2} \\
-\tau_{v} & u_{v}-u_{l}
\end{array}\right)\left(\begin{array}{c}
-A_{v} \\
0
\end{array}\right) .
$$

If the following condition holds

$$
\left(u_{g}-u_{l}\right)^{2}-c_{g}^{2} \neq 0, \quad \text { and } \quad\left(u_{v}-u_{l}\right)^{2}-c_{v}^{2} \neq 0,
$$

then the right eigenvectors are linearly independant and they span $\mathbb{R}^{10}$. 
We now investigate the nature of the waves, Riemann invariants and jump conditions.

Proposition 3 (Nature of the coupling wave). Fields associated with eigenvalues $\lambda_{3}=\lambda_{4}=u_{l}$ are linearly degenerated and admit the following Riemann invariants

$$
\begin{aligned}
& I_{3,4}^{1}(\mathbf{W})=u_{l}, \quad I_{3,4}^{2}(\mathbf{W})=s_{g}, \quad I_{3,4}^{3}(\mathbf{W})=s_{v}, \\
& I_{3,4}^{4}(\mathbf{W})=m_{v}\left(u_{l}-u_{v}\right), \quad I_{3,4}^{5}(\mathbf{W})=m_{g}\left(u_{l}-u_{g}\right), \\
& I_{3,4}^{6}(\mathbf{W})=\varepsilon_{v}+\frac{p_{v}}{\rho_{v}}+\frac{1}{2}\left(u_{v}-u_{l}\right)^{2}, \\
& I_{3,4}^{7}(\mathbf{W})=\varepsilon_{g}+\frac{p_{g}}{\rho_{g}}+\frac{1}{2}\left(u_{g}-u_{l}\right)^{2}, \\
& I_{3,4}^{8}(\mathbf{W})=\sum_{k \in \mathbb{K}} \alpha_{k} p_{k}+\sum_{k=g, v} m_{k}\left(u_{k}-u_{l}\right)^{2} .
\end{aligned}
$$

The proof is straightforward but tedious. To check the Riemann invariants $I_{3,4}^{j}$, $j=1, \ldots, 8$, one has to verify that

$$
\nabla_{\mathbf{W}} I_{3,4}^{j}(\mathbf{W}) \cdot r_{3}(\mathbf{W})=\nabla_{\mathbf{W}} I_{3,4}^{j}(\mathbf{W}) \cdot r_{4}(\mathbf{W})=0 .
$$

Similarly one has the following characterization of the remaining fields.

Proposition 4 (Riemann invariants). The field associated with the eigenvalue $\lambda_{1}=$ $u_{g}$ is linearly degenerated with the Riemann invariants

$$
\begin{aligned}
I_{1}^{1}(\mathbf{W})=u_{g}, & I_{1}^{2}(\mathbf{W})=p_{g}, \quad I_{1}^{3}(\mathbf{W})=\alpha_{g}, \\
I_{1}^{4,5}(\mathbf{W})=\rho_{k}, & I_{1}^{6,7}(\mathbf{W})=u_{k}, \quad I_{1}^{8,9}(\mathbf{W})=p_{k},
\end{aligned}
$$

with $k=l, v$. Similar results hold for $\lambda_{2}=u_{v}$ with

$$
\begin{aligned}
I_{2}^{1}(\mathbf{W})=u_{v}, & I_{2}^{2}(\mathbf{W}) \quad=p_{v}, \quad I_{2}^{3}(\mathbf{W})=\alpha_{g}, \\
I_{2}^{4,5}(\mathbf{W})=\rho_{k}, & I_{2}^{6,7}(\mathbf{W})=u_{k}, \quad I_{2}^{8,9}(\mathbf{W})=p_{k},
\end{aligned}
$$

with $k=l, g$.

Fields associated with eigenvalues $u_{k} \pm c_{k}, k \in \mathbb{K}$ (that is $\lambda_{5,6}, \lambda_{7,8}, \lambda_{9,10}$ ) are genuinely non linear with Riemann invariants

$$
\begin{aligned}
& I_{5,6,7,8,9,10}^{1}(\mathbf{W})=s_{k}, \quad I_{5,6,7,8,9,10}^{2}(\mathbf{W})=u_{k} \mp \int_{0}^{\rho} \frac{c_{k}\left(r, s_{k}\right)}{r} d r \\
& I_{5,6,7,8,9,10}^{3}(\mathbf{W})=\alpha_{g}, \\
& I_{5,6,7,8,9,10}^{4}(\mathbf{W})=\rho_{k^{\prime}}, \quad I_{5,6,7,8,9,10}^{5}(\mathbf{W})=u_{k^{\prime}}, \quad I_{5,6,7,8,9,10}^{6}(\mathbf{W})=p_{k^{\prime}} \\
& I_{5,6,7,8,9,10}^{7}(\mathbf{W})=\rho_{k^{\prime \prime}}, \quad I_{5,6,7,8,9,10}^{8}(\mathbf{W})=u_{k^{\prime \prime}}, \quad I_{5,6,7,8,9,10}^{9}(\mathbf{W})=p_{k^{\prime \prime}}
\end{aligned}
$$

with $k^{\prime} \neq k^{\prime \prime} \neq k$.

As the genuinely non-linear fields are concerned, i.e. the $p$-fields with $p=$ $5, \ldots, 10$, we assume the following jump relations across an isolated discontinuity 
travelling at speed $\sigma$

$$
\begin{aligned}
& {\left[\alpha_{k}\right]=0,} \\
& {\left[m_{k}\left(u_{k}-\sigma\right)\right]=0,} \\
& {\left[m_{k} u_{k}\left(u_{k}-\sigma\right)+\alpha_{k} p_{k}\right]=0,} \\
& {\left[m_{k} E_{k}\left(u_{k}-\sigma\right)+\alpha_{k} p_{k} u_{k}\right]=0,} \\
& {\left[\rho_{k^{\prime}}\right]=0, \quad\left[\rho_{k^{\prime \prime}}\right]=0,} \\
& {\left[u_{k^{\prime}}\right]=0, \quad\left[u_{k^{\prime \prime}}\right]=0,} \\
& {\left[p_{k^{\prime}}\right]=0, \quad\left[p_{k^{\prime \prime}}\right]=0,}
\end{aligned}
$$

with $k, k^{\prime}, k^{\prime \prime} \in \mathbb{K}, k^{\prime} \neq k, k^{\prime \prime} \neq k$, where the notation [.] refers to the jump between the states $R$ and $L$ separated by the discontinuity, namely $[g]=g_{R}-g_{L}$. We emphasize that the $p$-Riemann invariants and the jump relations for the linearly degenerated $p$-fields, $p=1,2,3,4$, coincide. Thus appart from the coupling wave associated with $\lambda_{3}=\lambda_{4}=u_{l}$, the jump relations are those of a single-phase Euler system.

Remark 1. The eigenstructure of the system (39)-(40) and its jump relations are a key point to build solutions to the Riemann problem of the system. To do so, starting from a left state $\mathbf{Y}_{L}$, we need to fix a real value through each genuinely nonlinear field and for each linearly degenerated field associated with $\lambda_{i}, i=1,2$ with velocities $u_{g}, u_{v}$ respectively. For the coupling wave associated to $\lambda_{3}=\lambda_{4}=u_{l}$, we need to fix the right void fraction of the gas $\alpha_{g, R}$ and the right density of the liquid $\rho_{l, R}$. Eventually one deduces the corresponding right state $\mathbf{Y}_{R}$.

2.3. Admissible structure of the source terms. The purpose of this section is to properly define admissible source terms toward the thermodynamical equilibrium. More specifically one has to exhibit the constraints on the mechanical transfer term $\Phi_{g}(\mathbf{Y})$, the mass transfer terms $\Gamma_{k}(\mathbf{Y})$, the momentum source terms $\mathcal{D}(\mathbf{Y})$ and the total energy source terms $\mathcal{B}(\mathbf{Y})$ such that the entropy growth criterion holds for smooth solutions.

We introduce the following notations while skipping the dependency in $\mathbf{Y}$ for sake of readability.

(a) The mass transfer terms $\Gamma_{k}$ is decomposed as

$$
\Gamma_{k}=\sum_{k^{\prime} \neq k \in \mathbb{K}} \tilde{\Gamma}_{k k^{\prime}},
$$

where the dyadic contributions cancel by conservation of the total mass:

$$
\tilde{\Gamma}_{k k^{\prime}}+\tilde{\Gamma}_{k^{\prime} k}=0 .
$$

Keep in mind that if the gas phase is inert, then $\Gamma_{g}=0$ and relations (49) and (50) hold true for $k, k^{\prime} \in\{l, v\}$.

(b) The source terms in the momentum equations are decomposed into a mass transfer contribution and velocity contribution, namely

$$
\mathcal{D}_{k}=\sum_{k^{\prime} \neq k \in \mathbb{K}} \tilde{d}_{k k^{\prime}}+\sum_{k^{\prime} \neq k \in \mathbb{K}} \tilde{v}_{k k^{\prime}} \tilde{\Gamma}_{k k^{\prime}}
$$


with the symmetry and antisymmetry assumptions

$$
\begin{aligned}
& \tilde{v}_{k k^{\prime}}=\tilde{v}_{k^{\prime} k}, \\
& \tilde{d}_{k k^{\prime}}+\tilde{d}_{k^{\prime} k}=0 .
\end{aligned}
$$

(c) The energy transfer terms are decomposed into a thermal contribution, a velocity contribution and a mass transfer contribution, as follow

$$
\mathcal{B}_{k}=\sum_{k^{\prime} \neq k \in \mathbb{K}} \tilde{\Psi}_{k k^{\prime}}+\sum_{k^{\prime} \neq k \in \mathbb{K}} \tilde{v}_{k k^{\prime}} \tilde{d}_{k k^{\prime}}+\sum_{k^{\prime} \neq k \in \mathbb{K}} \tilde{H}_{k k^{\prime}} \tilde{\Gamma}_{k k^{\prime}}
$$

with the symmetry assumption

$$
\tilde{H}_{k k^{\prime}}=\tilde{H}_{k^{\prime} k} .
$$

and the antisymmetry assumption

$$
\tilde{\Psi}_{k k^{\prime}}+\tilde{\Psi}_{k^{\prime} k}=0 .
$$

Using the decompositions (49)-(55), the right hand side $R H S_{\eta}$ of the entropy identity (23)-(26) now reads

$$
R H S_{\eta}=R H S_{\eta}^{\Phi}+R H S_{\eta}^{u}+R H S_{\eta}^{T}+R H S_{\eta}^{\mu},
$$

where $R H S_{\eta}^{\Phi}$ is given in (36) and the remain the drag effect term and the thermal term are

$$
\begin{aligned}
R H S_{\eta}^{u} & =\sum_{k \in \mathbb{K}} a_{k}\left(\sum_{k^{\prime} \neq k \in \mathbb{K}} \tilde{v}_{k k^{\prime}} \tilde{d}_{k k^{\prime}}-\left(\sum_{k^{\prime} \neq k \in \mathbb{K}} \tilde{d}_{k k^{\prime}}\right) u_{k}\right), \\
R H S_{\eta}^{T} & =\sum_{k \in \mathbb{K}} a_{k}\left(\sum_{k^{\prime} \neq k \in \mathbb{K}} \tilde{\Psi}_{k k^{\prime}}\right) .
\end{aligned}
$$

One may rewrite the source terms $R H S_{\eta}^{T}$ in term of dyadic bonds, using the antisymmetry assumption (55) of the quantities $\tilde{\Psi}_{k k^{\prime}}, k \neq k^{\prime} \in \mathbb{K}$. It yields

$$
R H S_{\eta}^{T}=\frac{1}{2} \sum_{k^{\prime} \neq k \in \mathbb{K}}\left(a_{k}-a_{k^{\prime}}\right) \tilde{\Psi}_{k k^{\prime}}
$$

where the prefactor $1 / 2$ ensures to count the dyadic bounds only once. If one assumes that

$$
\tilde{v}_{k k^{\prime}}=\delta_{k k^{\prime}} u_{k}+\left(1-\delta_{k k^{\prime}}\right) u_{k^{\prime}}, \quad \text { with } \delta_{k k^{\prime}}+\delta_{k^{\prime} k}=1,
$$

then, using the antisymmetry property (52) of the quantities $d_{k k^{\prime}}, k \neq k^{\prime}$, the source term $R H S_{\eta}^{u}$ reads

$$
\begin{aligned}
R H S_{\eta}^{u} & =\sum_{k \in \mathbb{K}} a_{k}\left(\sum_{k^{\prime} \neq k \in \mathbb{K}}\left(\tilde{v}_{k k^{\prime}}-u_{k}\right) \tilde{d}_{k k^{\prime}}\right) \\
& =\frac{1}{2} \sum_{k^{\prime} \neq k \in \mathbb{K}}\left[a_{k}\left(1-\delta_{k k^{\prime}}\right)+a_{k^{\prime}} \delta_{k k^{\prime}}\right]\left(u_{k^{\prime}}-u_{k}\right) \tilde{d}_{k k^{\prime}} \\
& =\frac{1}{2} \sum_{k^{\prime} \neq k \in \mathbb{K}}\left[a_{k} \delta_{k^{\prime} k}+a_{k^{\prime}} \delta_{k k^{\prime}}\right]\left(u_{k^{\prime}}-u_{k}\right) \tilde{d}_{k k^{\prime}} .
\end{aligned}
$$

From the source term expressions (36), (58), (60) one deduces easily the constraints imposed on the source terms in order to ensure the entropy growth criterion. 
Proposition 5 (Admissible source terms). The source terms comply with the entropy inequality

$$
\partial_{t} \eta(\mathbf{Y})+\partial_{x} f_{\eta}(\mathbf{Y}) \geq 0
$$

for smooth solutions of (5)-(8) with minimal entropy dissipation $A\left(\mathbf{Y}, \partial_{x} \mathbf{Y}\right)=0$, if, for any dyadic bond $k^{\prime} \neq k \in \mathbb{K}$, it holds

$$
\begin{aligned}
& \Phi_{g}(\mathbf{Y})\left(p_{v}+p_{g}-p_{l}\right) \geq 0 \\
& \left(a_{k}-a_{k^{\prime}}\right) \tilde{\Psi}_{k k^{\prime}} \geq 0, \\
& \left(a_{k} \delta_{k^{\prime} k}+a_{k^{\prime}} \delta_{k k^{\prime}}\right)\left(u_{k^{\prime}}-u_{k}\right) \tilde{d}_{k k^{\prime}} \geq 0 .
\end{aligned}
$$

Moreover if $\tilde{v}_{k k^{\prime}}=\frac{1}{2}\left(u_{k}+u_{k^{\prime}}\right)$ and $\tilde{H}_{k k^{\prime}}=\frac{u_{k} u_{k^{\prime}}}{2}$, it holds also

$$
\tilde{\Gamma}_{k k^{\prime}}\left(a_{k^{\prime}} \mu_{k^{\prime}}-a_{k} \mu_{k}\right) \geq 0 .
$$

Proof. Relations (62) are deduced by construction. We focus now on the mass transfer terms. To do so we explicit the term $R H S^{\mu}$ using (56), (26), (58) and (60)

$$
\begin{aligned}
R H S_{\eta}^{\mu} & =-\frac{1}{2} \sum_{k^{\prime} \neq k \in \mathbb{K}} \tilde{\Gamma}_{k k^{\prime}}\left(a_{k} \mu_{k}-a_{k}^{\prime} \mu_{k}^{\prime}\right) \\
& +\sum_{k \in \mathbb{K}} a_{k}\left(\sum_{k^{\prime} \neq k \in \mathbb{K}}\left(\tilde{H}_{k k^{\prime}}-u_{k} \tilde{v}_{k k^{\prime}}+\frac{u_{k}^{2}}{2}\right) \tilde{\Gamma}_{k k^{\prime}}\right) .
\end{aligned}
$$

If $\tilde{v}_{k k^{\prime}}=\frac{1}{2}\left(u_{k}+u_{k^{\prime}}\right)$ and $\tilde{H}_{k k^{\prime}}=\frac{u_{k} u_{k^{\prime}}}{2}$, then the second term of (64) cancels and $R H S_{\eta}^{\mu} \geq 0$ that is (63) holds true.

One observes that the mechanical relaxation source term has to comply with the Dalton's law on the gaseous pressures.

Remark 2. The relaxation source terms are implicitly weighted by relaxation positive time scales. In practice the quantities $\tilde{d}_{k k^{\prime}}, \tilde{\Psi}_{k k^{\prime}}$ and $\Gamma_{k k^{\prime}}$, corresponding to the drag terms, the interfacial energy transfer and the mass transfer, are taken from the two-phase flow literature. The modelling of physically relevant relaxation time scales is a topic in itself and is not addressed in the present paper. We refer for instance to [13] for the modelling of pressure relaxation time scales.

Remark 3. In Proposition 5, the condition (63) guarantees that the relaxation with respect to mass transfer is in agreement with the second principle of thermodynamics. It assumes that the relaxation acts between any couple of phases $k, k^{\prime}$. Nonetheless, considering a three-phase mixture of an inert gas, a liquid and its vapor, the mass transfer only occurs between the liquid and its vapor. Hence, the condition (63) of Proposition 5 should be reduced to $k=l$ and $k^{\prime}=v$ (and vice versa). Hence $\tilde{\Gamma}_{k k^{\prime}}=0$ for $k, k^{\prime}=g$.

2.4. Symmetrization. The symmetrization of the two-velocity two-pressure BaerNunziato-like models has been investigated in [6], the case of barotropic two-phase flow being addressed in [24]. Here we adapt the proofs to exhibit that the system convective system associated to (5)-(8) admits a symmetric form.

The system is symmetrizable if there exists a $C^{1}$-diffeomorphism from $\mathbb{R}^{10}$ to $\mathbb{R}^{10} g: \mathbf{U} \rightarrow \mathbf{W}$ (where the "conservative" variables $\mathbf{U}$ and the state vector $\mathbf{W}$ 
are defined respectively in (14) and (38)), a symmetric positive definite matrix $\mathbb{D}(\mathbf{W}) \in \mathbb{R}^{10 \times 10}$ and a symmetric matrix $\mathbb{Q}(\mathbf{W}) \in \mathbb{R}^{10 \times 10}$ such that the smooth solutions of the system satisfy

$$
\mathbb{D}(\mathbf{W}) \partial_{t} \mathbf{W}+\mathbb{Q}(\mathbf{W}) \partial_{x} \mathbf{W}=0 .
$$

Recall that $\mathbf{W}=\left(\alpha_{g}, p_{g}, u_{g}, p_{v}, u_{v}, p_{l}, u_{l}, s_{g}, s_{v}, s_{l}\right)$ is defined in (38). We introduce the following symmetric matrix

$$
\mathbb{D}(W)=\left(\begin{array}{ccccc}
A_{0} & \mathbf{e}_{g}^{\top} & \mathbf{e}_{v}^{\top} & \mathbf{e}_{l}^{\top} & \mathbb{O}_{1 \times 3} \\
\mathbf{e}_{g} & \mathbf{D}_{g} & \mathbb{O}_{2 \times 2} & \mathbb{O}_{2 \times 2} & \mathbb{O}_{2 \times 3} \\
\mathbf{e}_{v} & \mathbb{O}_{2 \times 2} & \mathbf{D}_{v} & \mathbb{O}_{2 \times 2} & \mathbb{O}_{2 \times 3} \\
\mathbf{e}_{l} & \mathbb{O}_{2 \times 2} & \mathbb{O}_{2 \times 2} & \mathbf{D}_{l} & \mathbb{O}_{2 \times 3} \\
\mathbb{O}_{3 \times 1} & \mathbb{O}_{3 \times 2} & \mathbb{O}_{3 \times 2} & \mathbb{O}_{3 \times 2} & \mathbb{1}_{3 \times 3}
\end{array}\right),
$$

where $\mathbb{O}_{n \times m}$ denotes the null matrix of size $n \times m, \mathbb{1}_{n \times m}$ is the identity matrix of size $n \times m$ and $\mathbf{D}_{k}$ is given by

$$
\mathbf{D}_{k}=\left(\begin{array}{cc}
1 & 0 \\
0 & \rho_{k}^{2} c_{k}^{2}
\end{array}\right), \quad k \in \mathbb{K}
$$

and $\mathbf{e}_{k}, k \in \mathbb{K}$, is the solution of the linear system

$$
\left(\begin{array}{cc}
u_{k}-u_{l} & \tau_{k} \\
\rho_{k} c_{k}^{2} & u_{k}-u_{l}
\end{array}\right) \mathbf{e}_{k}=\mathbf{D}_{k} \mathbf{f}_{k},
$$

with $\mathbf{f}_{k}=\left(A_{k}, 0\right)^{\top}$ for $k=g, v$ and $\mathbf{f}_{l}=\left(0, A_{l}\right)^{\top}$. One needs to determine a suitable value of the nonnegative scalar $A_{0}$.

Theorem 1. The system (39) is symmetrizable with

- the symmetric positive definite matrix $\mathbb{D}(\mathbf{W}) \in \mathbb{R}^{10 \times 10}$ defined by (66), (67) and (68)

- the symmetric matrix $\mathbb{Q}(\mathbf{W})=\mathbb{D}(\mathbf{W}) \mathbb{B}(\mathbf{W}) \in \mathbb{R}^{10 \times 10}, \mathbb{B}(\mathbf{W})$ being defined in (40)-(41),

if and only if the nonnegative scalar $A_{0}$ complies with

$$
\begin{aligned}
\left(\rho_{l} \rho_{g} \rho_{v} c_{l} c_{g} c_{v}\right)^{2} & \left(A_{0}-\mathbf{e}_{g, 1}^{2}-\mathbf{e}_{v, 1}^{2}-\mathbf{e}_{l, 1}^{2}\right. \\
& \left.-\mathbf{e}_{g, 2}^{2} /\left(\rho_{g} c_{g}\right)^{2}-\mathbf{e}_{v, 2}^{2} /\left(\rho_{v} c_{v}\right)^{2}-\mathbf{e}_{l, 2}^{2} /\left(\rho_{l} c_{l}\right)^{2}\right)>0,
\end{aligned}
$$

where $\mathbf{e}_{k, i}$ refers to the coefficient $i=1,2$ of the vector $\mathbf{e}_{k}$.

Proof. Exploiting the block structures of the matrices $\mathbb{D}(\mathbf{W})$ and $\mathbb{B}(\mathbf{W})$, one can check that the matrix $\mathbb{Q}(\mathbf{W})$ is symmetric. First observe that the convection matrix $\mathbb{B}(\mathbf{W})$ may be written into the following block shape

$$
\mathbb{B}(\mathbf{Y})=\left(\begin{array}{ccccc}
u_{l} & \mathbb{O}_{1 \times 2} & \mathbb{O}_{1 \times 2} & \mathbb{O}_{1 \times 2} & \mathbb{O}_{1 \times 3} \\
\mathbf{f}_{g} & \mathbf{C}_{g} & \mathbb{O}_{2 \times 2} & \mathbb{O}_{2 \times 2} & \mathbb{O}_{2 \times 3} \\
\mathbf{f}_{v} & \mathbb{O}_{2 \times 2} & \mathbf{C}_{v} & \mathbb{O}_{2 \times 2} & \mathbb{O}_{2 \times 3} \\
\mathbf{f}_{l} & \mathbb{O}_{2 \times 2} & \mathbb{O}_{2 \times 2} & \mathbf{C}_{l} & \mathbb{O}_{2 \times 3} \\
\mathbb{O}_{3 \times 2} & \mathbb{O}_{3 \times 2} & \mathbb{O}_{3 \times 2} & \mathbb{O}_{3 \times 2} & \mathbf{C}_{u}
\end{array}\right),
$$

with the matrices $\mathbf{C}_{k}$ and $\mathbf{C}_{u}$ such that

$$
\mathbf{C}_{k}=\left(\begin{array}{cc}
u_{k} & \rho_{k} c_{k}^{2} \\
\tau_{k} & u_{k}
\end{array}\right), \quad k \in \mathbb{K}, \quad \mathbf{C}_{u}=\operatorname{diag}\left(u_{g}, u_{v}, u_{l}\right) \in \mathbb{R}^{3 \times 3} .
$$


The matrix $\mathbb{Q}(\mathbf{W})=\mathbb{D}(\mathbf{W}) \mathbb{B}(\mathbf{W})$ is symmetric if and only if (68) holds. Note that the system (68) is invertible unless the resonance condition (42) occurs. The matrix $\mathbb{D}(\mathbf{W})$ is a symmetric positive definite matrix as soon as its upper $7 \times 7$-block

$$
\left(\begin{array}{cccc}
A_{0} & \mathbf{e}_{g}^{\top} & \mathbf{e}_{v}^{\top} & \mathbf{e}_{l}^{\top} \\
\mathbf{e}_{g} & \mathbf{D}_{g} & \mathbb{D}_{2 \times 2} & \mathbb{D}_{2 \times 2} \\
\mathbf{e}_{v} & \mathbb{D}_{2 \times 2} & \mathbf{D}_{v} & \mathbb{D}_{2 \times 2} \\
\mathbf{e}_{l} & \mathbb{D}_{2 \times 2} & \mathbb{D}_{2 \times 2} & \mathbf{D}_{l}
\end{array}\right),
$$

is symmetric positive definite. Thus one has to prove that the minor determinants are nonnegative. The 7 minor determinants are the following:

$$
\begin{aligned}
& \delta_{1}=A_{0}, \\
& \delta_{2}=A_{0}-\mathbf{e}_{g, 1}^{2}, \\
& \delta_{3}=\rho_{g}^{2} c_{g}^{2}\left(A_{0}-\mathbf{e}_{g, 1}^{2}\right)-\mathbf{e}_{g, 2}^{2}, \\
& \delta_{4}=\delta_{3}-\rho_{g}^{2} c_{g}^{2} \mathbf{e}_{v, 1}^{2}, \\
& \delta_{5}=\rho_{v}^{2} c_{v}^{2} \delta_{4}-\rho_{g}^{2} c_{g}^{2} \mathbf{e}_{v, 2}^{2} \\
& \delta_{6}=\delta_{5}-\left(\rho_{g} \rho_{v} c_{g} c_{v}\right)^{2} \mathbf{e}_{l, 1}^{2}, \\
& \delta_{7}=\rho_{l}^{2} c_{l}^{3} \delta_{6}-\left(\rho_{g} \rho_{v} c_{g} c_{v}\right)^{2} \mathbf{e}_{l, 2}^{2} .
\end{aligned}
$$

One observes that $\delta_{7}>0$ implies that $\delta_{6}>0$ and so on until $\delta_{1}>0$. Hence a sufficient and necessary condition to ensure that $\mathbb{D}(\mathbf{W})$ is (symmetric) positive definite is $\delta_{7}>0$ which coincides with (69).

As a consequence of the Kato's theorem [26], starting from an admissible solution satisfying (69), there exits a local in time smooth solution to the Cauchy problem. Assuming that the resonance condition (42) does not occur, the time of existence corresponds to the time of blow up of the $L^{\infty}$ norm of the spatial derivative of the solutions.

\section{Conclusion}

We address in this paper the derivation of a Bear-Nunziato-like model for a compressible three-phase flow. The particularity of the three-phase mixture relies on the miscibility constraints between each phase: the liquid phase is immiscible with the two gaseous phases which are miscible. It leads to the equality of the gaseous void fractions. This feature allows to recover that the gaseous phases satisfy the Dalton's law. Besides we also prove that the interfacial pressures are uniquely defined by enforcing a physically relevant entropy inequality for smooth solutions of the whole system. The derivation of the model and the relaxation source terms agree with the second law of thermodynamics. The homogeneous model is hyperbolic and symmetrizable, leading to a local existence result. Note that the source terms are defined up to the determination of reliable relaxation time scales. We also provide in Appendix B a restriction to the barotropic case.

In future works it would be interesting to address the numerical approximation of the three-phase model. To do so it seems mandatory to use an accurate numerical scheme such as the one developed in [32]. Eventually the model should be used to simulate a realistic three-phase test case for instance the Reactivity Initiated Accident. Actually the latter phenomenon involves droplets of molten solid, 
water under its liquid and vapor phases and fission gases. Hence it would be necessary to extend our derivation to the four-phase case accounting for proper volumic constraints.

\section{Acknowledgments}

This work has received the financial support from the ANR MoHyCon.

\section{Appendix A. Phasic equations}

Restricting to regular solutions, manipulations of the system (5)- (8) allow to write the nonconservative phasic equations on the densities $\rho_{k}$, the velocities $u_{k}$ and the momentum $u_{k}^{2} / 2$, the internal energies $m_{k} \varepsilon_{k}$ and the pressures $p_{k}$, with $k \in \mathbb{K}$. They read

$$
\partial_{t} \rho_{k}+u_{k} \partial_{x} \rho_{k}+\rho_{k} \partial_{x} u_{k}+\frac{\rho_{k}}{\alpha_{k}}\left(-v_{i}(\mathbf{Y})+u_{k}\right) \partial_{x} \alpha_{k}=\frac{\Gamma_{k}(\mathbf{Y})-\rho_{k} \Phi_{k}(\mathbf{Y})}{\alpha_{k}}
$$

$$
\partial_{t} u_{k}+u_{k} \partial_{x} u_{k}+\frac{1}{m_{k}}\left(\partial_{x}\left(\alpha_{k} p_{k}\right)+\pi_{k}(\mathbf{Y}) \partial_{x} \alpha_{g}\right)=\frac{\mathcal{D}_{k}(\mathbf{Y})-u_{k} \Gamma_{k}(\mathbf{Y})}{m_{k}}
$$

$$
\begin{aligned}
\partial_{t}\left(m_{k} \frac{u_{k}^{2}}{2}\right) & +\partial_{x}\left(m_{k} \frac{u_{k}^{2}}{2} u_{k}+\alpha_{k} u_{k} p_{k}\right)-\alpha_{k} p_{k} \partial_{x} u_{k}+u_{k} \pi_{k}(\mathbf{Y}) \partial_{x} \alpha_{g} \\
& =\mathcal{D}_{k}(\mathbf{Y}) u_{k}-\Gamma_{k}(\mathbf{Y}) u_{k}^{2} / 2,
\end{aligned}
$$

$$
\begin{aligned}
\partial_{t}\left(m_{k} \varepsilon_{k}\right)+\partial_{x}\left(m_{k} \varepsilon_{k} u_{k}\right)+\alpha_{k} p_{k} \partial_{x} u_{k}+\pi_{k}(\mathbf{Y})\left(v_{i}(\mathbf{Y})-u_{k}\right) \partial_{x} \alpha_{g} \\
\quad=\mathcal{B}_{k}(\mathbf{Y})-\mathcal{D}_{k}(\mathbf{Y}) u_{k}+\Gamma_{k}(\mathbf{Y}) \frac{u_{k}^{2}}{2}+\pi_{k}(\mathbf{Y}) \Phi_{g}(\mathbf{Y}) \\
\partial_{t} p_{k}+u_{k} \partial_{x} p_{k}+\rho_{k} c_{k}^{2} \partial_{x} u_{k} \\
+\left(\frac{\partial \varepsilon_{k}}{\partial p_{k}}\right)^{-1} \frac{1}{m_{k}}\left(\pi_{k}(\mathbf{Y}) \partial_{x} \alpha_{g}+p_{k} \partial_{x} \alpha_{k}\right)\left(v_{i}(\mathbf{Y})-u_{k}\right) \\
+\frac{\rho_{k} c_{k}^{2}}{\alpha_{k}}\left(u_{k}-v_{i}(\mathbf{Y})\right) \partial_{x} \alpha_{k} \\
=\left(\frac{\partial \varepsilon_{k}}{\partial p_{k}}\right)^{-1} \frac{1}{m_{k}}\left(\mathcal{B}_{k}(\mathbf{Y})-\mathcal{D}_{k}(\mathbf{Y}) u_{k}\right. \\
\left.+\Gamma_{k}(\mathbf{Y})\left(\frac{u_{k}^{2}}{2}-\varepsilon_{k}-\rho_{k} \frac{\partial \varepsilon_{k}}{\partial \rho_{k}}\right)+\pi_{k}(\mathbf{Y}) \Phi_{g}(\mathbf{Y})+\rho_{k}^{2} \frac{\partial \varepsilon_{k}}{\partial \rho_{k}} \Phi_{k}(\mathbf{Y})\right)
\end{aligned}
$$


Then one derives the equation on the phasic entropies $s_{k}$, which are functions of $\rho_{k}$ and $p_{k}$.

$$
\begin{aligned}
\partial_{t} s_{k} & +u_{k} \partial_{x} s_{k}+\frac{\partial s_{k}}{\partial p_{k}}\left(\frac{\partial \varepsilon_{k}}{\partial p_{k}}\right)^{-1}\left(\pi_{k}(\mathbf{Y}) \partial_{x} \alpha_{g}+p_{k} \partial_{x} \alpha_{k}\right)\left(v_{i}(\mathbf{Y})-u_{k}\right) / m_{k} \\
& =\frac{\partial s_{k}}{\partial \rho_{k}}\left(\frac{\Gamma_{k}(\mathbf{Y})-\rho_{k} \Phi_{k}(\mathbf{Y})}{\alpha_{k}}\right) \\
& +\frac{a_{k}}{m_{k}}\left(\mathcal{B}_{k}(\mathbf{Y})-\mathcal{D}_{k}(\mathbf{Y}) u_{k}+\Gamma_{k}(\mathbf{Y})\left(\frac{u_{k}^{2}}{2}-\varepsilon_{k}-\rho_{k} \frac{\partial \varepsilon_{k}}{\partial \rho_{k}}\right)\right. \\
& \left.+\pi_{k}(\mathbf{Y}) \Phi_{g}(\mathbf{Y})+\rho_{k}^{2} \frac{\partial \varepsilon_{k}}{\partial \rho_{k}} \Phi_{k}(\mathbf{Y})\right) .
\end{aligned}
$$

This way one gets the evolution equation (23) for the mixture entropy $\eta$.

\section{Appendix B. Derivation of a BAROtropic MOdEL}

Consider the homogeneous model associated to three-phase non equilibrium model (5)-(8). We focus on the equations (75) satisfied by the phasic entropies $s_{k}, k \in \mathbb{K}$, see Appendix A. If the interfacial velocity is $v_{i}(\mathbf{Y})=v_{l}$, then one can check that the phasic entropies are transported with velocities $u_{k}$

$$
\partial_{t} s_{k}+u_{k} \partial_{x} s_{k}=R H S_{\eta}(\mathbf{Y}) \text {. }
$$

Assuming that entropy production terms are small enough, one may consider the approximation:

$$
\partial_{t} s_{k}+u_{k} \partial_{x} s_{k}=R H S_{\eta}(\mathbf{Y})
$$

which admits the particular solution

$$
s_{k}\left(p_{k}, \rho_{k}\right)=s_{k, 0} .
$$

We now fix

$$
p_{k}=p_{k}\left(\rho_{k}, s_{k, 0}\right), \quad k \in \mathbb{K},
$$

and plug these pressures into the non equilibrium model (5)-(8) (with relaxation terms). It reduces to the following barotropic model

$$
\begin{aligned}
& \partial_{t} \alpha_{g}+v_{i}\left(\mathbf{Y}^{\mathbf{b}}\right) \partial_{x} \alpha_{g}=\Phi_{g}\left(\mathbf{Y}^{\mathbf{b}}\right), \\
& \partial_{t} m_{k}+\partial_{x}\left(m_{k} u_{k}\right)=0, \quad k \in \mathbb{K}, \\
& \partial_{t}\left(m_{l} u_{l}\right)+\partial_{x}\left(m_{l} u_{l}^{2}+\alpha_{l} p_{l}\right)+\Pi\left(\mathbf{Y}^{b}\right) \partial_{x} \alpha_{g}=D_{l}\left(\mathbf{Y}^{\mathbf{b}}\right), \\
& \partial_{t}\left(M u_{g}\right)+\partial_{x}\left(M u_{g}^{2}+\mathcal{P}\right)-\Pi\left(\mathbf{Y}^{b}\right) \partial_{x} \alpha_{g}=\mathcal{D}_{g}\left(Y^{b}\right),
\end{aligned}
$$

where

$$
\begin{aligned}
M & =\alpha_{g}\left(\rho_{g}+\rho_{v}\right), \\
\mathcal{P} & =\alpha_{g}\left(p_{g}+p_{v}\right) .
\end{aligned}
$$

The state vector is now

$$
\mathbf{Y}^{b}=\left\{\alpha_{g}, \rho_{l}, \rho_{g}, \rho_{v}, u_{l}, u_{g}\right\}
$$

Observe here that for this particular model we will assume that the gaseous phases $k=g, v$ evolve with the same velocity $u_{g}$. The source terms $\Phi_{g}(Y)$ and $\mathcal{D}_{g}(Y), k=l, g$, describe the relaxation toward the mechanical equilibrium and 
the drag force. The relaxation terms and the interfacial terms should cancel when considering the mean flow, hence one has

$$
\mathcal{D}_{l}\left(\mathbf{Y}^{b}\right)=-\mathcal{D}_{g}\left(\mathbf{Y}^{b}\right) .
$$

Once more we need to define the interfacial velocity $v_{i}\left(\mathbf{Y}^{b}\right)$ in $(79)$ and the interfacial pressure $\Pi\left(\mathbf{Y}^{b}\right)$ in (81) and (82). To do so, we derive the equation satisfied by the mixture entropy. Since we focus on the barotropic case, the internal phasic energies are now $e_{k}=e_{k}\left(\rho_{k}\right), k \in \mathbb{K}$, such that

$$
e_{k}^{\prime}\left(\rho_{k}\right)=\frac{p_{k}\left(\rho_{k}\right)}{\rho_{k}^{2}},
$$

where $p_{k}=p_{k}\left(\rho_{k}\right)$ is the phasic barotropic pressure law (we skip in the sequel the dependency on $s_{0, k}$ for sake of readability).

The mixture entropy reads

$$
\eta\left(\mathbf{Y}^{b}\right)=\sum_{k \in \mathbb{K}} \alpha_{k} \rho_{k} \mathcal{E}_{k},
$$

where $\mathcal{E}_{k}=u_{k}^{2} / 2+e_{k}\left(\rho_{k}\right)$ denote the phasic energies.

The mixture entropy is governed by the following identity

$$
\begin{aligned}
\partial_{t} \eta\left(\mathbf{Y}^{b}\right) & +\partial_{x} f_{\eta}\left(\mathbf{Y}^{b}\right)+\mathcal{A}\left(\mathbf{Y}^{b}\right) \partial_{x} \alpha_{g} \\
& =\Phi_{g}\left(\mathbf{Y}^{b}\right)\left(p_{l}-\left(p_{g}+p_{v}\right)\right)+\mathcal{D}_{l}\left(\mathbf{Y}^{b}\right) u_{l}+\mathcal{D}_{g}\left(\mathbf{Y}^{b}\right) u_{g},
\end{aligned}
$$

where the entropy flux is

$$
f_{\eta}\left(\mathbf{Y}^{b}\right)=\sum_{k \in \mathbb{K}} m_{k} \mathcal{E}_{k} u_{k}+\sum_{k \in \mathbb{K}} \alpha_{k} u_{k} p_{k}
$$

and

$$
\mathcal{A}\left(\mathbf{Y}^{b}\right)=-\Pi\left(\mathbf{Y}^{b}\right)\left(u_{g}-u_{l}\right)+\left(p_{g}+p_{v}\right)\left(u_{g}-v_{i}\left(\mathbf{Y}^{b}\right)\right)-p_{l}\left(u_{l}-v_{i}\left(\mathbf{Y}^{b}\right)\right) .
$$

We assume again that the velocity $v_{i}\left(\mathbf{Y}^{b}\right)$ is a convex combination of the velocities $u_{l}$ and $u_{g}$

$$
v_{i}(Y)=\beta\left(\mathbf{Y}^{b}\right) u_{g}+\left(1-\beta\left(\mathbf{Y}^{b}\right)\right) u_{l},
$$

with $\beta\left(\mathbf{Y}^{b}\right) \in[0,1]$.

Proposition 6 (Closure law for $\left.\Pi\left(\mathbf{Y}^{b}\right)\right)$. Smooth solutions of system (79)-(82) comply with the constraint $\mathcal{A}\left(\mathbf{Y}^{b}\right)=0$ if and only if

$$
\Pi\left(\mathbf{Y}^{b}\right)=\beta\left(\mathbf{Y}^{b}\right) p_{l}+\left(1-\beta\left(\mathbf{Y}^{b}\right)\right)\left(p_{g}+p_{v}\right) .
$$

Proposition 7 (Entropy inequality). We assume that the source terms comply with

$$
\left\{\begin{array}{l}
\Phi_{g}\left(\mathbf{Y}^{b}\right)\left(p_{l}-\left(p_{g}+p_{v}\right)\right) \leq 0, \\
\mathcal{D}_{l}\left(\mathbf{Y}^{b}\right)\left(u_{l}-u_{g}\right) \leq 0
\end{array}\right.
$$

Then smooth solutions of system (79)-(82) satisfy the following inequality

$$
\partial_{t} \eta\left(\mathbf{Y}^{b}\right)+\partial_{x} f_{\eta}\left(\mathbf{Y}^{b}\right) \leq 0
$$

assuming $\mathcal{A}\left(\mathbf{Y}^{b}\right)=0$. 
Proposition 8 (Hyperbolicity). The convective system associated with the homogeneous part of system (79)-(82) is hyperbolic. It admits six eigenvalues

$$
\begin{aligned}
& \lambda_{1}=v_{i}\left(\mathbf{Y}^{b}\right), \quad \lambda_{2}=u_{g}, \\
\lambda_{3,4}= & u_{l} \pm c_{l}, \quad \lambda_{5,6}=u_{g} \pm \tilde{c},
\end{aligned}
$$

where

$$
\tilde{c}^{2}=\frac{m_{v} c_{v}^{2}+m_{g} c_{g}^{2}}{M}
$$

setting $c_{k}^{2}=p^{\prime}\left(\rho_{k}\right)$ for $k=g, v$.

Proposition 9 (Interface velocity). Consider the interface velocity $v_{i}\left(\mathbf{Y}^{b}\right)$ given by the convex combination (91) with $\beta\left(\mathbf{Y}^{b}\right) \in[0,1]$. If $\beta\left(\mathbf{Y}^{b}\right)=0$, or $\beta\left(\mathbf{Y}^{b}\right)=1$ or $\beta\left(\mathbf{Y}^{b}\right)=M /\left(M+m_{l}\right)$ then the field associated with $\lambda_{1}\left(\mathbf{Y}^{b}\right)=v_{i}\left(\mathbf{Y}^{b}\right)$ is linearly degenerated.

\section{REFERENCES}

[1] M. R. Baer and J. W. Nunziato. A two phase mixture theory for the deflagration to detonation (ddt) transition in reactive granular materials. Int. J. Multiphase Flow, 12(6):861-889, 1986.

[2] T. Barberon and P. Helluy. Finite volume simulation of cavitating flows. Computers and Fluids, 34(7):832-858, 2005.

[3] D. Bothe and W. Dreyer. Continuum thermodynamics of chemically reacting fluid mixtures. Acta Mech., 226(6):1757-1805, 2015. Available from: https://doi.org/10.1007/ s00707-014-1275-1.

[4] Y. Chen, Y.-F. Deng, J. Glimm, G. Li, Q. Zhang, and D. H. Sharp. A renormalization group scaling analysis for compressible two-phase flow. Phys. Fluids A, 5(11):2929-2937, 1993. Available from: https://doi.org/10.1063/1.858701, doi:10.1063/1.858701.

[5] F. Coquel, J.-M. Hérard, K. Saleh, and N. Seguin. A class of two-fluid two-phase flow models. 42nd AIAA Fluid Dynamics Conference and Exhibit, Fluid Dynamics and Co-located Conferences, 2012. Available from: https://doi.org/10.2514/6.2012-3356.

[6] F. Coquel, J.-M. Hérard, K. Saleh, and N. Seguin. Two properties of two-velocity twopressure models for two-phase flows. Commun. Math. Sci., 12(3):593-600, 2014. Available from: https://doi.org/10.4310/CMS.2014.v12.n3.a10.

[7] S. Dellacherie. Relaxation schemes for the multicomponent Euler system. M2AN Math. Model. Numer. Anal., 37(6):909-936, 2003. Available from: http://dx.doi.org/10.1051/ m2an:2003061, doi:10.1051/m2an:2003061.

[8] D. A. Drew and S. L. Passman. Theory of multicomponent fluids, volume 135 of Applied Mathematical Sciences. Springer-Verlag, New York, 1999. Available from: https://doi.org/ 10.1007/b97678.

[9] D.A. Drew. Mathematical modeling of two-phase flow. Ann. Rev. Fluid Mech., 15:261-291, 1983.

[10] P. Embid and M. Baer. Mathematical analysis of a two-phase continuum mixture theory. Contin. Mech. Thermodyn., 4(4):279-312, 1992. Available from: https://doi.org/10.1007/ BF01129333.

[11] T. Flåtten and H. Lund. Relaxation two-phase flow models and the subcharacteristic condition. Math. Models Methods Appl. Sci., 21(12):2379-2407, 2011. Available from: http: //dx.doi.org/10.1142/S0218202511005775.

[12] T. Gallouët, J.-M. Hérard, and N. Seguin. Numerical modeling of two-phase flows using the two-fluid two-pressure approach. Math. Models Methods Appl. Sci., 14(5):663700, 2004. Available from: http://dx.doi.org/10.1142/S0218202504003404, doi:10.1142/ S0218202504003404.

[13] S. Gavrilyuk. The structure of pressure relaxation terms: the one-velocity case. Technical report, EDF, H-I83-2014-0276-EN, 2014.

[14] S. Gavrilyuk and R. Saurel. Mathematical and numerical modeling of two-phase compressible flows with micro-inertia. J. Comput. Phys., 175(1):326-360, 2002. Available from: http: //dx.doi.org/10.1006/jcph.2001.6951. 
[15] V. Guillemaud. Modelling and numerical simulation of two-phase flows using the two-fluid two-pressure approach. Theses, Université de Provence - Aix-Marseille I, March 2007. Available from: https://tel.archives-ouvertes.fr/tel-00169178.

[16] E. Han, M. Hantke, and S. Müller. Efficient and robust relaxation procedures for multicomponent mixtures including phase transition. J. Comput. Phys., 338:217-239, 2017. Available from: http://dx.doi.org/10.1016/j.jcp.2017.02.066.

[17] M. Hantke and S. Müller. Analysis and simulation of a new multi-component two-phase flow model with phase transitions and chemical reactions. Quart. Appl. Math., 76(2):253-287, 2018. Available from: https://doi.org/10.1090/qam/1498.

[18] M. Hantke and S. Müller. Closure conditions for a one temperature non-equilibrium multicomponent model of baer-nunziato type. ESAIM Proc, to appear, 2019.

[19] J.-M. Hérard. A three-phase flow model. Math. Comput. Modelling, 45(5-6):732-755, 2007. Available from: http://dx.doi.org/10.1016/j.mcm.2006.07.018, doi:10.1016/j. mcm.2006.07.018.

[20] J.-M. Hérard. Un modèle hyperbolique diphasique bi-fluide en milieu poreux. Comptes Rendus Mécanique, 742(8):623-683, 2008. Available from: http://www.sciencedirect.com/science/ article/pii/S1631-0721(08)00111-3, doi:http://dx.doi.org/10.1016/j.crme.2008.06. 005.

[21] J.-M. Hérard. A class of compressible multiphase flow models. C. R. Math. Acad. Sci. Paris, 354(9):954-959, 2016. Available from: http://dx.doi.org/10.1016/j.crma.2016.07.004, doi:10.1016/j.crma.2016.07.004.

[22] O. Hurisse and L. Quibel. A homogeneous model for compressible three-phase flows involving heat and mass transfer. ESAIM Proc, to appear, 2019.

[23] K. Hutter and K. Jöhnk. Continuum methods of physical modeling. Springer-Verlag, Berlin, 2004. Continuum mechanics, dimensional analysis, turbulence. Available from: https://doi. org/10.1007/978-3-662-06402-3.

[24] David Iampietro. Contribution to the simulation of low-velocity compressible two-phase flows with high pressure jumps using homogeneous and two-fluid approaches. Theses, AixMarseille Université, November 2018. Available from: https://tel.archives-ouvertes.fr/ tel-01919156.

[25] A.K. Kapila, R. Menikoff, J.B. Bdzil, S.F. Son, and D.S. Stewart. Two-phase modelling of ddt in granular materials: reduced equations. Phys. Fluids, 13:3002-3024, 2001.

[26] T. Kato. The Cauchy problem for quasi-linear symmetric hyperbolic systems. Arch. Rational Mech. Anal., 58(3):181-205, 1975. Available from: https://doi.org/10.1007/BF00280740.

[27] H. Lochon. Modelling and simulation of steam-water transients using the two-fluid approach. Theses, Aix Marseille Université, October 2016. Available from: https://tel. archives-ouvertes.fr/tel-01379453.

[28] H. Lochon, F. Daude, P. Galon, and J.-M. Hérard. Computation of fast depressurization of water using a two-fluid model: revisiting Bilicki modelling of mass transfer. Comput. \& Fluids, 156:162-174, 2017. Available from: https://doi.org/10.1016/j.compfluid.2017.07.008.

[29] H. Mathis. A thermodynamically consistent model of a liquid-vapor fluid with a gas. M2AN Math. Model. Numer. Anal., 2018. Available from: DOI:https://doi.org/10.1051/m2an/ 2018044, doi:10.1051/m2an/2018044.

[30] S. Müller, M. Hantke, and P. Richter. Closure conditions for non-equilibrium multi-component models. Contin. Mech. Thermodyn., 28(4):1157-1189, 2016. Available from: http://dx.doi. org/10.1007/s00161-015-0468-8.

[31] M. Pelanti, K.-M. Shyue, and T. Flåtten. A numerical model for three-phase liquid-vapor-gas flows with relaxation processes. Hyp2016.

[32] K. Saleh. A relaxation scheme for a hyperbolic multiphase flow model. Part I: barotropic eos. working paper or preprint, March 2018. Available from: https://hal.archives-ouvertes. fr/hal-01737681.

EDF R\&D, 6, Quiai Watier, 78400 Chatou, France

E-mail address: jean-marc.herard@edf.fr

Université de Nantes, CNRS, Laboratoire de Mathématiques Jean Leray (LMJL UMR 6629), F-44000 NANTES, France

E-mail address: helene.mathis@univ-nantes.fr 